\title{
Chaotic temporal variations in cosmic masers
}

\author{
Abraham C.-L. Chian, Erico L. Rempel \\ National Institute for Space Research - INPE, P. O. Box 515, São José \\ dos Campos-SP 12201-970, Brazil
}

Félix A. Borotto

Universidad de Concepción, Departamento de Física, Concepción, Chile

\begin{abstract}
Electron cyclotron maser emission is an accepted physical mechanism for generating coherent planetary and stellar radio emissions. Observational data has indicated evidence of nonlinear and chaotic temporal variability in some cosmic masers such as solar microwave spikes. The nonlinear and chaotic characteristics of cosmic masers can be attributed to plasma turbulence, such as Alfvén chaos, embedded in the emission region. We report a chaos theory of Alfvén waves which can account for chaotic acceleration of electrons in the source region of cosmic masers. Two types of Alfvén intermittency are identified: PomeauManneville intermittency and crisis-induced intermittency. Since Alfvén waves may be responsible for accelerating electrons that emit maser radiations, the chaotic dynamics of Alfvén waves may be the origin of chaotic time variations of cosmic masers. Hence, we suggest that Alfvén intermittency may cause intermittent temporal fluctuations which can be observed in cosmic masers.
\end{abstract}

Coherent emissions in the cosmos can be due to either cyclotron maser emission (Wu and Lee 1979; Melrose 1991, 1994) or plasma emission (Chian and Alves 1988; Chian et al. 1997, 2000). Observational evidence of electron cyclotron maser emission (ECME) has been obtained for the auroral kilometric radiation (AKR) from the Earth, the decametric radio emission from Jupiter and analogous emissions from other giant planets of the solar system. In addition, ECME can also be the source of solar microwave spike bursts and very bright radio emissions from some flare stars. A direct amplification of the extraordinary mode and ordinary mode AKR emission is possible when a population of suprathermal auroral electrons, reflected by the magnetic mirror effect, possess a loss-cone distribution. The cyclotron maser instability operates if the electrons satisfy a relativistic cyclotron resonance condition, which produces the bunching of electrons and the consequent coherent radiation. The relativistic effect is crucial in the amplification of ECME, without which the radiation will be damped even if the electrons have a loss-cone distribution.

Ultralow-frequency magnetohydrodynamic waves, such as Alfvén waves, have been observed in the emission regions of ECME (Chian and Oliveira, 1994; Chian et al. 2000). These Alfvén waves can be responsible for electron acceleration along the field lines, thus playing a fundamental role in the generation 
of ECME. It is likely that the observed dynamic features of ECME are related to the dynamics of Alfvén waves in the emission regions of ECME. Data analysis of solar microwave spike bursts demonstrated the presence of chaos. The quantitative characterization of a large number of decimetric pulsation events and millisecond spikes during solar flares gave evidence that they typically have their origin in chaotic attractors of low dimensionality (Kurth et al. 1991), which renders support for the occurrence of deterministic chaos either in the maser emission mechanism or the particle acceleration mechanism (Aschwanden et al. 1994). In particular, temporal intermittency is frequently found in the nonlinear dynamic properties of solar radio emissions such as narrowband spikes (Isliker and Benz 1994).

Evidently, the study of nonlinear dynamical evolution of Alfvén waves is essential for a proper understanding of electron acceleration in the source region of cosmic masers. In particular, the question of how a nonlinear Alfvén wave evolves into Alfvén chaos must be addressed. The spatiotemporal dynamics of a nonlinear Alfvén wave propagating along an ambient magnetic field in the $x$ direction is governed by the derivative nonlinear Schrödinger equation

$$
\partial_{t} b+\alpha \partial_{x}\left(|b|^{2} b\right)-i(\mu+i \eta) \partial_{x}^{2} b=S(b, x, t)
$$

where $b=b_{y}+i b_{z}$ is the complex transverse magnetic field normalized to the constant ambient magnetic field $B_{0}$, time $t$ is normalized to $\omega_{c i}^{-1}$ (where $\omega_{c i}=$ $e B_{0} / m_{i}$ is the ion cyclotron frequency), space $x$ is normalized to $c_{A} / \omega_{c} i$ (where $c_{A}=B_{0} /\left(\mu_{0} \rho_{0}\right)^{1 / 2}$ is the Alfvén velocity), $\alpha=1 /[4(1-\beta)], \beta=c_{S}^{2} / c_{A}^{2}$ (where $c_{S}=\left(P_{0} \gamma \rho_{0}\right)^{1 / 2}$ is the acoustic velocity), $\mu=1 / 2$, and $\eta$ is the dissipative scale length. The external driving force $S(b, x, t)=A \exp (i k \phi)$ is a monochromatic left-hand circularly polarized wave with a wave phase $\phi=x-V t$ where $V$ is a constant wave velocity. By defining $k$ to be real, we consider the driver to be non-growing and undamped.

For stationary waves with $b=b(\phi)$, the first integral of Eq. (1) yields a set of ordinary differential equations

$$
\begin{gathered}
\dot{b}_{y}-\nu \dot{b}_{z}=\partial H / \partial b_{z}+a \cos \theta \\
\dot{b}_{z}+\nu \dot{b}_{y}=-\partial H / \partial b_{y}+a \sin \theta \\
\dot{\theta}=\Omega \\
H=\left(b^{2}-1\right)^{2} / 4-(\lambda / 2)(\vec{b}-\hat{y})^{2},
\end{gathered}
$$

where the dot denotes derivative with respect to the "temporal" variable $\tau=$ $\alpha b_{0}^{2} \phi / \mu, b \rightarrow b / b_{0}$ (where $b_{0}$ is an integration constant), $\theta=\Omega \phi, \Omega=\mu k /\left(\alpha b_{0}^{2}\right)$, $a=A /\left(\alpha b_{0}^{2} k\right), \nu=\eta / \mu, \lambda=-1+V /\left(\alpha b_{0}^{2}\right)$, and $\alpha>0$ (i.e., $\beta<1$ ) is assumed. In the absence of a driver $(a=0)$ the dimension of Eqs. (2)-(5) reduces to two and all solutions are regular representing periodic Alfvén waves, Alfvén solitons and Alfvén shocks, respectively. In the presence of a driver $(a \neq 0)$, the dimension of Eqs. (2)-(5) increases to three, making possible chaotic solutions.

Through an analysis of the bifurcation diagram and Poincaré maps (Chian et al. 1998) of the numerical solutions of Eqs. (2)-(5) (with $\Omega=-1, \nu=0.02$, 

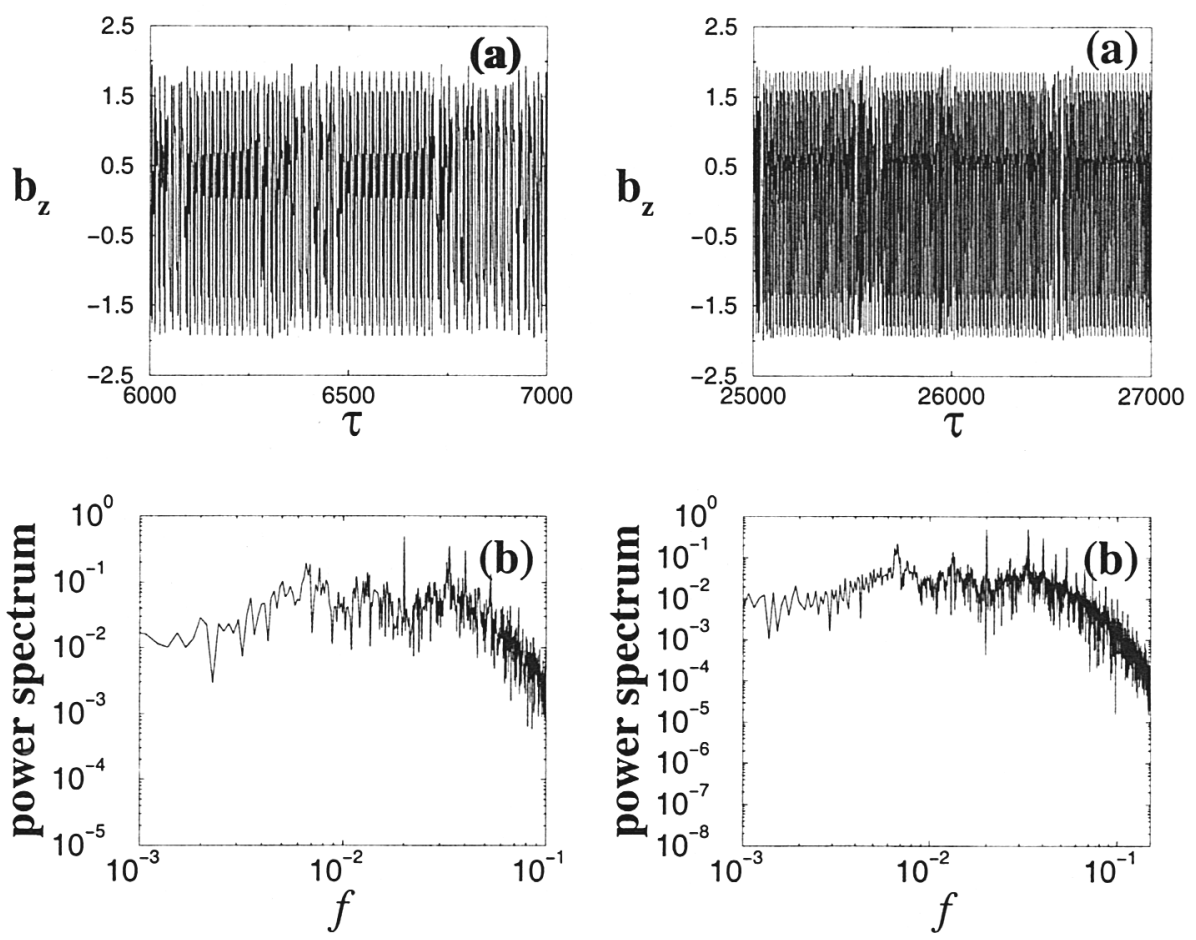

Figure 1. Example of the Pomeau-Manneville intermittency for $\mathrm{a}=0.3213795$. (a) Temporal series of $b_{z} ;(\mathrm{b})\left|b_{z}\right|^{2}$ as a function of frequency $f$.

Figure

2. Example of the crisis-induced intermittency for $\mathrm{a}=0.33029$. (a) temporal series of $b_{z}$; (b) $\left|b_{z}\right|^{2}$ as a function of frequency $f$.

$\lambda=1 / 4$ and $\beta<1$ ), we identified two types of Alfvén intermittency: PomeauManneville intermittency (Manneville and Pomeau 1979) and crisis-induced intermittency (Grebogi and Ott 1983). The Pomeau-Manneville intermittency is characterized by a time series containing nearly periodic laminar phases which are randomly interrupted by chaotic bursts, as exemplified by Figure 1a. The type-I Pomeau-Manneville intermittency occurs when a dynamical system is close to a saddle-node bifurcation arising from the coalescence of pairs of stable and unstable periodic orbits. The power spectrum for the times series of Figure $1 \mathrm{a}$ is shown in Figure 1b. The crisis-induced intermittency is characterized by a time series containing weakly chaotic phases which are randomly interrupted by strongly chaotic bursts (Grebogi and Ott 1983), as depicted in Figure 2a. This intermittency is associated with a type of chaotic transition called interior crisis, which occurs when an unstable periodic orbit (or its invariant stable manifolds) of the system collides with a chaotic attractor. The power spectrum for the times series of Figure 2a is shown in Figure 2b. 
In summary, we demonstrated that there are two types of Alfvén intermittency: Pomeau-Manneville intermittency and crisis-induced intermittency. Both types of Alfvén chaotic transitions involve an episodic switching between two different states of temporal behavior. In the Pomeau-Manneville intermittency, the time series of magnetic fluctuations switches intermittently between nearly periodic and chaotic states. These two types of Alfvén intermittency may induce chaotic acceleration of electrons in the source regions of cosmic masers, which in turn may be the origin of intermittency observable in the temporal fluctuations of cosmic maser emissions.

Acknowledgments. This research was supported by CNPq and FAPESP.

\section{References}

Aschwanden, M. J., Benz, A., O., Dennis, B. R., \& Kundu, M. R. 1994, ApJS, 90,631 .

Chian, A. C.-L. \& Alves, M. V. 1988, ApJ, 330, L77.

Chian, A. C.-L. \& Oliveira, L. P. L. 1994, A\&A, 286, L1.

Chian, A. C.-L., Abalde, J. R., Alves, M. V., \& Lopes, S. R. 1997, Solar Phys., $173,199$.

Chian, A. C.-L., Borotto, F. A., \& Gonzalez, W. D. 1998, ApJ, 503, 993.

Chian, A. C.-L., Borotto, F. A., Lopes, S. R., \& Abalde, J. R. 2000, Planetary Space Sci., 48, 9.

Grebogi, C. \& Ott, E. 1983, Physica, D7, 18.

Isliker, H. \& Benz, A. O. 1994, A\&A, 285, 663.

Kurth, J., Benz, A. O., \& Aschwanden, M. J. 1991, A\&A, 248, 270.

Manneville, P. \& Pomeau, Y. 1979, Phys. Lett. A, 75, 1.

Melrose, D. B. 1991, Ann. Rev. Astron. Astrophy., 11, 227.

Melrose, D.B. 1994, Space Sci. Rev., 68, 159.

Wu, C. S. \& Lee, L. C. 1979, ApJ, 230, 621. 